\title{
A SAMPLING THEOREM FOR ANALYTIC FUNCTIONS
}

\section{J. L. SCHIFF AND W. J. WALKER}

ABSTRaCt. An analogue to the Shannon sampling theorem is obtained for an analytic function sampled on a circle.

1. Introduction. Let $f$ be analytic on a domain $\Omega$ and let $D$ be a disk centered at $z_{0}$ with $\bar{D} \subset \Omega$. The sampling theorem presented here gives a surprising formula to recapture $f$ exactly from its values at $z_{0}$ and at a countable set of points on $\partial D$. The expansion obtained for $f$ is valid in the largest open disk center $z_{0}$ which is contained in $\Omega$.

Without loss of generality we take $z_{0}=0$ and suppose that $f$ has the Taylor expansion

$$
f(z)=\sum_{m=0}^{\infty} a_{m} z^{m}=\sum_{m=0}^{\infty} \frac{f^{(m)}(0)}{m !} z^{m}, \quad|z|<R .
$$

The theorem gives a precise formula for each $a_{m}$ (and hence for $f$ ) constructed from the countable set of values of $f$. The Shannon sampling theorem recently surveyed in [1] gives a formula to recapture a certain class of analytic function from its values at a countable set of points on the real axis. In the practical application of a sampling theorem only a finite set of data is sampled and the formula is truncated. The example in $\$ 3$ demonstrates how the theorem on the disk can give a very precise approximation with a small number of sample points.

2. The sampling theorem. In order to describe the method, we need the Möbius function $\mu$, which is defined as follows: Let $j$ be a positive integer:

(i) If $j=1$, then $\mu(j)=1$.

(ii) $\mu(j)=0$ if there is a prime $p$ such that $p^{2} \mid j$.

(iii) If $j$ has the prime factorization $j=p_{1} p_{2} \cdots p_{\lambda}$ where the primes are distinct, then $\mu(j)=(-1)^{\lambda}$.

The proof of the sampling theorem will require the following lemmas.

LEMMA 1. The Möbius function $\mu$ has the property that

$$
\sum_{d \mid h} \mu(d)= \begin{cases}1 & \text { for } h=1 \\ 0 & \text { for } h>1\end{cases}
$$

Received by the editors January 2, 1986.

1980 Mathematics Subject Classification (1985 Revision). Primary 30B99; Secondary $30 \mathrm{E} 10$. 
Proof. See [3, p. 114].

The next lemma first appeared in the work of J. L. Walsh [4]. We include the proof for completeness.

LEMMA 2. Let $f$ be analytic in the disk $|z|<R$. Let $k$ be a positive integer and set $\omega=e^{2 \pi i / k}$. Also fix $\zeta,|\zeta|<R$, and define

$$
\begin{gathered}
h_{1}(\zeta)=f(\zeta)-f(0), \\
h_{k}(\zeta)=f(\zeta)+f(\omega \zeta)+f\left(\omega^{2} \zeta\right)+\cdots+f\left(\omega^{k-1} \zeta\right)-k f(0), \quad k \geqslant 2 .
\end{gathered}
$$

Then

$$
h_{k}(\zeta)=k\left[\frac{f^{(k)}(0)}{k !} \zeta^{k}+\frac{f^{(2 k)}(0)}{(2 k) !} \zeta^{2 k}+\cdots+\frac{f^{(n k)}(0)}{(n k) !} \zeta^{n k}+\cdots\right] .
$$

Proof. For $k=1$ the proof is immediate from the Taylor series for $f$. Now suppose $k \geqslant 2$ and that $n$ is a multiple of $k$. Then

$$
1+\omega^{n}+\omega^{2 n}+\cdots+\omega^{(k-1) n}=k .
$$

If $n$ is not a multiple of $k$, then

$$
1+\omega^{n}+\omega^{2 n}+\cdots+\omega^{(k-1) n}=\left(1-\omega^{k n}\right) /\left(1-\omega^{n}\right)=0 .
$$

The Taylor expansion

$$
f(z)=f(0)+\frac{f^{(1)}(0)}{1 !} z+\cdots+\frac{f^{(n)}(0)}{n !} z^{n}+\cdots
$$

can now be applied to each term of $h_{k}(\zeta)$. The coefficient of $\zeta^{n}$ is

$$
\left[1+\omega^{n}+\omega^{2 n}+\cdots+\omega^{(k-1) n}\right] \frac{f^{(n)}(0)}{n !}
$$

and the result follows by evaluating this expression.

We now fix an initial point $\zeta$ and define the set of points $S$ at which $f$ is to be sampled as follows:

(i) $0 \in S$.

(ii) For each positive integer $k$ let $\omega=e^{2 \pi i / k}$; then the $k$ equally spaced points $\zeta$, $\omega \zeta, \omega^{2} \zeta, \ldots, \omega^{k-1} \zeta$, belong to $S$.

If $f$ is given at each point of $S$, then $h_{k}(\zeta)$ is given for $k \geqslant 1$. For convenience we set $H_{k}(\zeta)=h_{k}(\zeta) / k$. We can now state the sampling theorem.

THEOREM 3. Suppose that $f$ is given at each point of the set $S$. Then for each positive integer $m, a_{m}=f^{(m)}(0) / m$ ! is determined exactly by the formula

$$
a_{m}=\frac{1}{\zeta^{m}} \sum_{i=1}^{\infty} \mu(i) H_{i m}(\zeta)
$$

Hence $f(z)=f(0)+\sum_{m=1}^{\infty} a_{m} z^{m}$ is determined exactly. 
Proof. We need only verify the formula for $a_{m}$. To do this we use Lemma 2 to expand each term on the right-hand side of (1) so that the right-hand side can be viewed as a double series:

$$
\begin{aligned}
& \frac{\mu(1)}{\zeta^{m}} H_{m}(\zeta)=\frac{\mu(1)}{\zeta^{m}}\left[\frac{f^{(m)}(0)}{m !} \zeta^{m}+\frac{f^{(2 m)}(0)}{(2 m) !} \zeta^{2 m}+\frac{f^{(3 m)}(0)}{(3 m) !} \zeta^{3 m}+\frac{f^{(4 m)}(0)}{(4 m) !} \zeta^{4 m}+\cdots\right], \\
& \frac{\mu(2)}{\zeta^{m}} H_{2 m}(\zeta)=\frac{\mu(2)}{\zeta^{m}}\left[+\frac{f^{(2 m)}(0)}{(2 m) !} \zeta^{2 m}+\frac{f^{(4 m)}(0)}{(4 m) !} \zeta^{4 m}+\cdots\right] \text {, }
\end{aligned}
$$

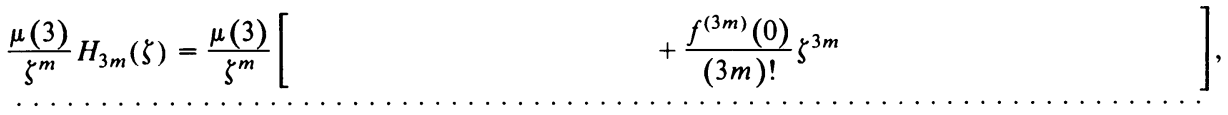

$$
\begin{aligned}
& \frac{\mu(j)}{\zeta^{m}} H_{j m}(\zeta)=\frac{\mu(j)}{\zeta^{m}}\left[\quad+\frac{f^{(j m)}(0)}{(j m) !} \zeta^{j m}\right] .
\end{aligned}
$$

We now compute coefficients by adding columns of this array. We denote the $j$ th column by $C_{j}$. Thus,

$$
\begin{aligned}
& C_{1}=\frac{\mu(1)}{\zeta^{m}} \frac{f^{(m)}(0)}{m !} \zeta^{m}=\frac{f^{(m)}(0)}{m !}=a_{m}, \\
& C_{2}=\frac{1}{\zeta^{m}}(\mu(1)+\mu(2)) \frac{f^{(2 m)}(0)}{(2 m !)} \zeta^{2 m}=0 .
\end{aligned}
$$

In general for $j \geqslant 2$ we can apply Lemma 1 to show

$$
C_{j}=\frac{1}{\zeta^{m}}\left(\sum_{d \mid j} \mu(d)\right) \frac{f^{(j m)}(0)}{(j m !)} \zeta^{j m}=0 .
$$

Then $\sum_{j=1}^{\infty} C_{j}=a_{m}$.

Thus the iterated series calculated by summing columns first converges to $a_{m}$. The iterated series calculated by summing rows first will also converge to $a_{m}$ if we can show that the double series is absolutely convergent. To do this we take the absolute value of each term in the array and sum columns. For $j \geqslant 2$ the $j$ th column becomes

$$
c_{j}=\frac{1}{\left|\zeta^{m}\right|}\left(\sum_{d \mid j}|\mu(d)|\right) \frac{\left|f^{(j m)}(0)\right|}{(j m) !}|\zeta|^{j m} \leqslant \frac{1}{\left|\zeta^{m}\right|} j \frac{\left|f^{(j m)}(0)\right|}{(j m) !}|\zeta|^{j m} .
$$

Then

$$
\sum_{j=1}^{\infty} c_{j} \leqslant \frac{1}{\left|\zeta^{m}\right|} \sum_{j=1}^{\infty} j \frac{\mid f^{(j m)}(0)}{(j m) !}|\zeta|^{j m}
$$

The right-hand series is convergent since $|\zeta|$ is less than the radius of convergence of the Taylor series. This completes the proof of the absolute convergence of the double series. Hence the iterated series, summing by rows first,

$$
\left(\zeta^{m}\right)^{-1} \sum_{i=1}^{\infty} \mu(i) H_{i m}(\zeta)
$$

also converges to $a_{m}$, which proves the theorem. 
3. Applications. In order to effectively approximate each Taylor coefficient, one must truncate the infinite series $\sum_{i=1}^{\infty} \mu(i) H_{i m}(\zeta)$ at some stage. In preliminary computer studies, we have used the preceding algorithm out to $i=10$, yielding

$$
a_{m} \simeq H_{1 m}(\zeta)-H_{2 m}(\zeta)-H_{3 m}(\zeta)-H_{5 m}(\zeta)+H_{6 m}(\zeta)-H_{7 m}(\zeta)+H_{10 m}(\zeta),
$$

$m=1,2,3, \ldots$. The number of points sampled can thus be kept relatively low, and yet highly precise estimates can still be achieved. For example, below is a table of estimates (for $i=10$ ) to the Taylor coefficients $a_{m}=1 / m$ ! of $f(z)=e^{z}, m=$ $1, \ldots, 5$.

\begin{tabular}{|l|l|l|}
\hline & \multicolumn{1}{|c|}{ Estimate } & \multicolumn{1}{|c|}{ Actual } \\
\hline$m=1$ & $1.00000012+0.00000018 i$ & 1 \\
$m=2$ & $0.49999991+0.00000001 i$ & 0.5 \\
$m=3$ & $0.16666642+0.00000003 i$ & $0.166 \ldots$ \\
$m=4$ & $0.04166676+0.00000001 i$ & $0.04166 \ldots$ \\
$m=5$ & $0.00833333-0.00000010 i$ & $0.00833 \ldots$ \\
\hline
\end{tabular}

The points sampled were on the unit circle, with the initial point $\zeta=1$.

If in a practical situation the value at the origin is unknown, then an estimate for it can be obtained from the values on the boundary circle using the mean value property (cf. [2]).

\section{REFERENCES}

1. J. R. Higgins, Five short stories about the cardinal series, Bull. Amer. Math. Soc. (N.S.) 12 (1985), 45-89.

2. H. Niederreiter, Quasi-monte carlo methods and pseudo-random numbers, Bull. Amer. Math. Soc. 84 (1978), 957-1041.

3. B. L. Van der Waerden, Modern algebra, vol. 1, Ungar, New York, 1953.

4. J. L. Walsh, A mean value theorem for polynomials and harmonic polynomials, Bull. Amer. Math. Soc. 42 (1936), 923-930.

Department of Mathematics and Statistics, University of Auckland, Auckland, New ZeaLAND 